\title{
Precision Marketing Strategies for Small and Medium-sized Clothing E-commerce Enterprises Based on Big Data
}

\author{
Yu-Chung Chang ${ }^{1 *}$
}

\begin{abstract}
Based on the big data analysis technology of small and medium-sized clothing ecommerce enterprises, this paper provides theoretical and practical methods for e-commerce enterprises to apply big data technology. The data sources come from questionnaires and online data grab. Through the Google online questionnaire survey, 252 valid questionnaires were received. Python is used to search valid reviews on Tmall and Internet with some keywords, and a total of 11,864 valid comments were obtained. We find that the reason why men buy clothes is as high as $68 \%$ because of the seasonal change, but only $31 \%$ of women buy clothes because of the seasonal change. The higher the average monthly income of women, the more attention is paid to whether the style of clothing design is suitable for themselves, and the more attention is paid to the service attitude of e-commerce enterprises. From decision tree analysis, the fit of clothing style is the most important factor affecting the shopping choice of customers. From the data of customer reviews, the most mentioned variables are clothing style, fashion, service attitude and price. The number of reviews and the sentiment analysis score on clothing style are all ranking first. The number of reviews and the sentiment analysis score on clothing fashion are all ranking second. We also proposes corresponding precision marketing strategies based on the results of big data analysis, such as the gender recommendation strategies, personalized recommendation strategies, differentiated marketing strategies, enterprise training and clothing design strategies.
\end{abstract}

Keywords: Big Data, E-commerce, Precision Marketing Strategies, Decision Tree, Sentiment Analysis

\section{Introduction}

In recent years, with the advancement of science and technology, the world has entered an era of informatization. E-commerce is booming, bringing people a faster and more convenient life. Now, online shopping has become an important channel for people's daily consumption, and the scale of consumption on e-commerce platforms has rapidly expanded. In the first quarter of 2020, China's online retail B2C market transaction scale reached 1,252.26 billion (RMB). The expansion of the consumption scale of e-commerce platforms has led to rapid growth in the amount of e-commerce data. Traditional marketing concepts and methods have been unable to meet the needs, and the marketing model of e-commerce has gradually changed. The technology for using big data analysis to achieve accurate ecommerce marketing came into being (Saidali et al., 2019).

Before big data technology was applied in the marketing, marketers usually used subjective awareness or market sample surveys to conduct marketing activities. Therefore, they were often not accurate enough and even caused great losses. Beyer \& Laney (2012)

\footnotetext{
${ }^{1}$ School of Information Science \& Technology, Xiamen University Tan Kah Kee College, 2933662796@qq.com * corresponding author
} 
pointed out that big data is a large amount of fast, multiple types of data, which requires efficient processing and analysis to obtain new knowledge that have not been find in the past to assist decision-making and show value (Beyer \& Laney, 2012). In this era of big data, systematically recording customers' consumption patterns and consumption habits has become feasible. If the recorded information is further analyzed by big data, it will be possible to launch more effective marketing and sales activities that meet customer needs.

With the development of cloud computing technology and network communication technology and the improvement of related infrastructure and services, the use of big data to make marketing measurable, and the realization of precise marketing is being realized step by step. Through data mining on big data, we can grasp valuable information to make judgments and decisions. Before data mining, it is necessary to understand consumers, and then accurately distinguish the market to ensure the effectiveness of market, product, and positioning. The internal departments of the company, such as market researchers, data analysts, information technology personnel, and marketing personnel, must also cooperate with each other to obtain accurate big data analysis results through data mining, and ultimately achieve a further increase in company profits.

Today, it is no longer regarded as an infringement on user privacy that e-commerce companies use big data to perform precision marketing by categorizing, analyzing, and mining the user's public information. Users have gradually accepted and enjoyed the convenience brought about by it. It is becoming more and more important to guide the company's marketing activities through data mining of e-commerce sales data. Various ecommerce giants have established professional departments to collect and use big data. Many large companies in the e-commerce platform have entrusted the e-commerce platform to conduct big data analysis, or set up a professional department to purchase data from the e-commerce platform for analysis.

However, for small and medium-sized e-commerce enterprises, the cost of entrusting an e-commerce platform for data analysis is higher. It is more costly and unnecessary to build a complete and effective big data analysis department. Purchase data from the ecommerce platform for analysis, the complexity and diversity of big data analysis requires a high level of expertise. As a result, there is a lack of research on the implementation and effect of big data precision marketing for small and medium-sized e-commerce enterprises.

In this paper, we will try to propose feasible methods to collect and analyze big data for small and medium-sized e-commerce enterprises, and propose precise marketing strategies based on the analysis results.

\section{Related Literature and the Big Data Precision Marketing Strategy 2.1 Consumer Behavior}

Consumer behavior refers to the various activities involved in people acquiring, consuming, and disposing of goods or services (Blackwell et al., 2001). Kotler (2000) defines consumer behavior as the entire process from when consumers are stimulated by external sources to making a purchase decision. This external stimulus enters the consumer's consciousness, and then the purchase decision based on the consumer's characteristics and decision-making process. Schiffman \& Kanuk (2014) believe that consumer behavior is the decision-making activities of consumers regarding products, services, purchase, use, evaluation, and disposal.

In order to meet different consumption goals, the formulation of corporate marketing strategies requires an understanding of consumer behavior patterns. By understanding consumers' age, income, preferences, time, price acceptance, and consumption habits, marketers can use marketing strategies to persuade different consumers to buy the products 
of the company.

\subsection{Integrated Marketing Strategy}

Kitchen et al. (2008) pointed out that integrated marketing is to use a database of consumer purchasing behavior and consumer insights to conduct marketing activities. Integrated marketing takes consumers as the center to further understand their consumption habits, enabling consumers to be identified and letting the cross-selling can be achieved to consumers. Rulac (1994) find that the traditional retail industry is being threatened by many emerging competitors, such as online shopping. The main reason is that the time that consumers can freely use in the business society has become less and less, so they will gradually reduce the frequency of going out for shopping. Rulac also pointed out that in a large-scale market research survey in 1990, it was found that the main considerations for consumers' preference for certain stores were product price, product selectivity, product quality, location of the store, and the quality of the service. It can be seen that the price level has a great influence on consumers' decision-making when shopping.

Reichheld \& Schefter $(1996 ; 2000)$ find that the most important thing for companies to retain customers is to establish more convenience in the service process. Some consumers want to be as simple as possible when shopping, and don't want to spend too much time searching for products, collecting information, and waiting for the shopping process. For example, fast food stores are a low-importance and high-convenience industry, so the simpler the shopping process can easier for customer patronize again.

\subsection{Big Data Precision Marketing Strategy}

Kusnetzky (2018) defines the big data as following: the amount of data involved is too large so that in a reasonable time we cannot manually retrieve, manage, process, and organize it into information that can be interpreted by humans. The characteristics description of big data ranges from the 3"V" of volume, variety, and velocity (Laney, 2001) to the 4"V" of volume, variety, velocity, and veracity (Hirzel et al., 2013) to the 5"V" of volume, variety, velocity, veracity, and value (Del Rowe, 2016).

Big data has been widely used in various fields in recent years, including the application of big data in tourism businesses, medical care, finance, e-commerce and sports. For instance, Brynjolfsson et al. (2011) find that the pieces of content uploaded to Facebook in some time are in the 30 billion while the value of big data for the healthcare industry is about 300 billion. By using big data analysis to understand the customers' behaviors, many travel businesses are taking advantage of channel-based distribution. The travel and tourism businesses are leveraging themselves with data-driven marketing, as they seek new customers and prospects (Camilleri, 2018).

Big data precision marketing strategy is a marketing method in a big data environment. It is based on massive data from multiple platforms and relies on data mining technology to accurately locate customers. On this basis, it establishes one-to-one communication with customers. The core of providing customers with personalized service experience is to accurately deliver product advertisements, information, and activities to the right people at the right time, through the right channels, and through the right methods.

You et al. (2005) proposed a precision marketing decision-making framework. Through this decision-making framework, we can identify the potential characteristics between different types of customers, so we can propose the appropriate precision marketing strategies. 


\subsection{Three-way Decisions Theory}

Three-way decisions theory (Yao, 2012) is the extension of traditional binary decision theory. Its ideological basis is derived from rough set and dominance based rough set theory (DRST). The main purpose is to interpret the positive domain, negative domain, and boundary domain of the rough set model as three decision results, namely acceptance, rejection, and delay. Through the semantic study of the positive, negative, and boundary domains of the collection, by calculating the probability and threshold of the object classification category. According to the different domains of the object, three decisions are made respectively to accept that the object belongs to the target class, reject the object to belong to the target class, and delay the decision whether the object belongs to the target class. The delayed decision-making requires further information collection and analysis, and then make corresponding decisions based on the new analysis results and the actual situation, that is, accept or reject.

Due to the uncertainty and incompleteness of information, it is often impossible to make a clear decision to accept or reject. Three-way decisions theory has one more delayed option than traditional binary decision theory. When the information is uncertain and insufficient to support acceptance or rejection, the third option should be adopted. Three-way decisions theory believes that delayed selection is also a decision. Therefore, the three-way decisions theory can effectively process and classify data, reduce errors and improve the accuracy of decision-making.

\subsection{Sentiment Analysis Technology}

Hatzivassiloglou \& McKeown (1997) proposed a method of judging sentimental orientation in 1997. If the positive and negative of a certain word in the same sentence is known, the positive and negative of another new word can be judged through different conjunction. But this method can only deal with syntactic structures containing conjunction, and the recognition patterns and processing objects are greatly restricted.

Riloff \& Wieber (2003) artificially developed a template and selected seed evaluation words, and iteratively obtained evaluation words of the noun part of speech. In addition to the tendency analysis of the vocabulary, it can also be analyzed according to the context to get the tendency of the sentence, including the distinction between subjectivity and objectivity of sentences, and judging the degree of praise and criticism of subjective sentences. They lexicalized the text to give part of speech, and manually review the word segmentation results to establish the subject vocabulary database, attribute vocabulary database, negative vocabulary database, neutral vocabulary database, and evaluation vocabulary database. After the word segmentation result of a new text is compared with the vocabulary databases, the positive and negative scores will be calculated according to the opinion extracted after the comparison, or the decision will be delayed, and the information will be collected and analyzed before the positive and negative scores are formed. It represents emotional score.

\section{Research Method}

\subsection{Data Sources}

The data in this paper comes from questionnaires and online data grab. The source of the questionnaire data is through the Google online questionnaire survey, combined with the form of offline questionnaire release, a total of 300 questionnaires were distributed and 252 valid questionnaires were received. The SPSS was then used for reliability analysis, and it was found that the reliability was greater than 0.7 , indicating that the data had a good internal correlation. 
This paper takes Tmall's small and medium-sized clothing enterprises as an example for research. In order to understand the sales situation of small and medium-sized clothing enterprises, python is used to search valid reviews on Tmall and Internet with keywords "men's clothing", “women's clothing", “children's clothing", "shirt", “dress", "skirt", "trousers", "outerwear" and "sportswear". We also search for relevant text with keyword attractive "fashion", "design", "price", "quality", "style", "advertisement" and "service attitude", to study the frequency and evaluation of these variables in customer reviews. After deducting the relevant comments of large-scale well-known brand clothing, a total of 11,864 valid comments were obtained.

\subsection{Tools for Big Data Processing}

In this paper, we use SPSS to test the reliability of the questionnaire. The CHAID decision tree is used to classify the selection factors of small and medium-sized clothing ecommerce consumers to buy clothes, and to find out the main factors of purchase and the next-level consideration factors. Through the data visualization tool, using K-Means cluster analysis and the presentation of data visualization, we can classify consumers who buy clothing according to the reasons for the purchase and establish a database of users of the same type for analysis and prediction.

\subsection{Big Data and Sentiment Analysis}

We combine the ternary decision theory and sentiment analysis technology to compare and interpret the collected text data. If we take "this product is very good" as an example, the "product" is an attribute word, "very" is an adverb word, and "good" is an adjective word. According to the positive and negative judgment, we can give weighted scores "product $(+1)^{*} \operatorname{good}(+1)=+1$ ". That is, the attribute word is multiplied by the evaluation word (adjective) to calculate the emotional score, and the positive score represents positive emotions, negative score represents negative emotions. Another example is "This product looks good at first glance". When the method of multiplying the attribute word with the evaluation word: "product $(+1) * \operatorname{good}(+1)=+1$ " meets the neutral word "at first glance", the decision will be delayed. If the follow-up text appears "the design is not fashionable and the service attitude is bad after purchase", then the subsequent information "not fashionable and bad" will be used to generate emotional scores "product $(+1)^{*}$ not fashionable and bad $(-1)=-1 "$.

After sentiment analysis and judgment of the collected text data, we calculate the total score of each variable to find out the main variables that small and medium-sized clothing enterprises are concerned or praised by customers, and propose precision marketing strategies based on these variables.

\section{Results and Discussion}

\subsection{Decision Tree Analysis}

The classification model based on CHAID decision tree analysis is shown in Figure 1. According to the generated decision tree for further analysis, the following results can be obtained:

(1)The reason why men buy clothes is as high as $68 \%$ because of the seasonal change, but only $31 \%$ of women buy clothes because of the seasonal change. Therefore, women buy clothes not only because of need. The higher the average monthly income of women, the more attention is paid to whether the style of clothing design is suitable for themselves, and the more attention is paid to the service attitude of e-commerce enterprises. 


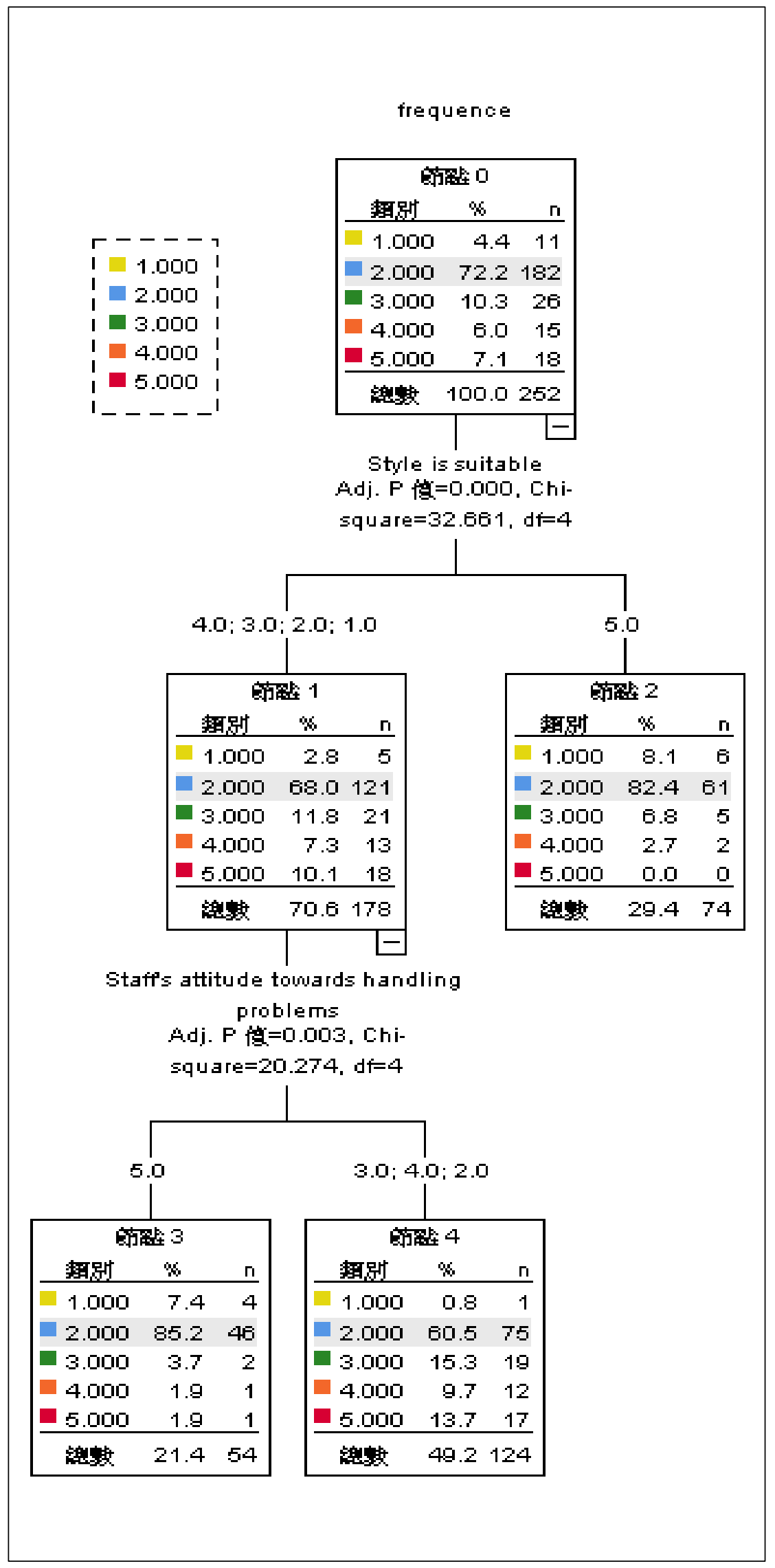

Figure 1. CHAID decision tree analysis 
(2)Ten factors are introduced into the rule. They are the frequency of purchase, attractive fashion design, the influence of demand, the influence of price, the clothing quality, the influence of advertisement, the influence of good reputation among customers, the style is suitable, the service attitude of employees and the average monthly income. As the first layer of decision tree, purchase frequency is the biggest classification factor affecting the shopping choice of customers in small and medium-sized clothing ecommerce enterprises. The fit of clothing style is the most important factor affecting the purchase. $70.6 \%$ of the buyers affected their purchase intention because of the appropriate style. The secondary reason affecting the purchase is the service attitude of small and medium-sized clothing e-commerce enterprises. There are $49.2 \%$ among the consumers who buy because of the fit of clothing style will be influenced by the service attitude of the small and medium-sized clothing e-commerce enterprises.

(3)According to the generation process of the decision tree, from the root node to each leaf node can be regarded as a classification rule, in which the feature index used is the main influencing factor of this category. In the decision tree of Figure 1, the classification: the frequency of buying clothes $\rightarrow$ the service attitude of small and medium-sized clothing e-commerce enterprises $\rightarrow$ attractive fashion design account for $34.7 \%$ of the total sample number, which means that the service attitude and attractive fashion design have a relatively large impact on people's purchase of clothing.

\subsection{Data Visualization of K-Means Cluster}

Import the variables to the visualization tool, including the frequency of purchase, attractive fashion design, the influence of demand, the influence of price, the clothing quality, the influence of advertisement, the influence of good reputation among customers, the style is suitable, the service attitude of employees and the average monthly income. Taking the result of decision tree analysis as an example, the 3D relationship graph among the three main factors as purchase frequency, suitability of styles and service attitude of employees is shown in Figure 2.

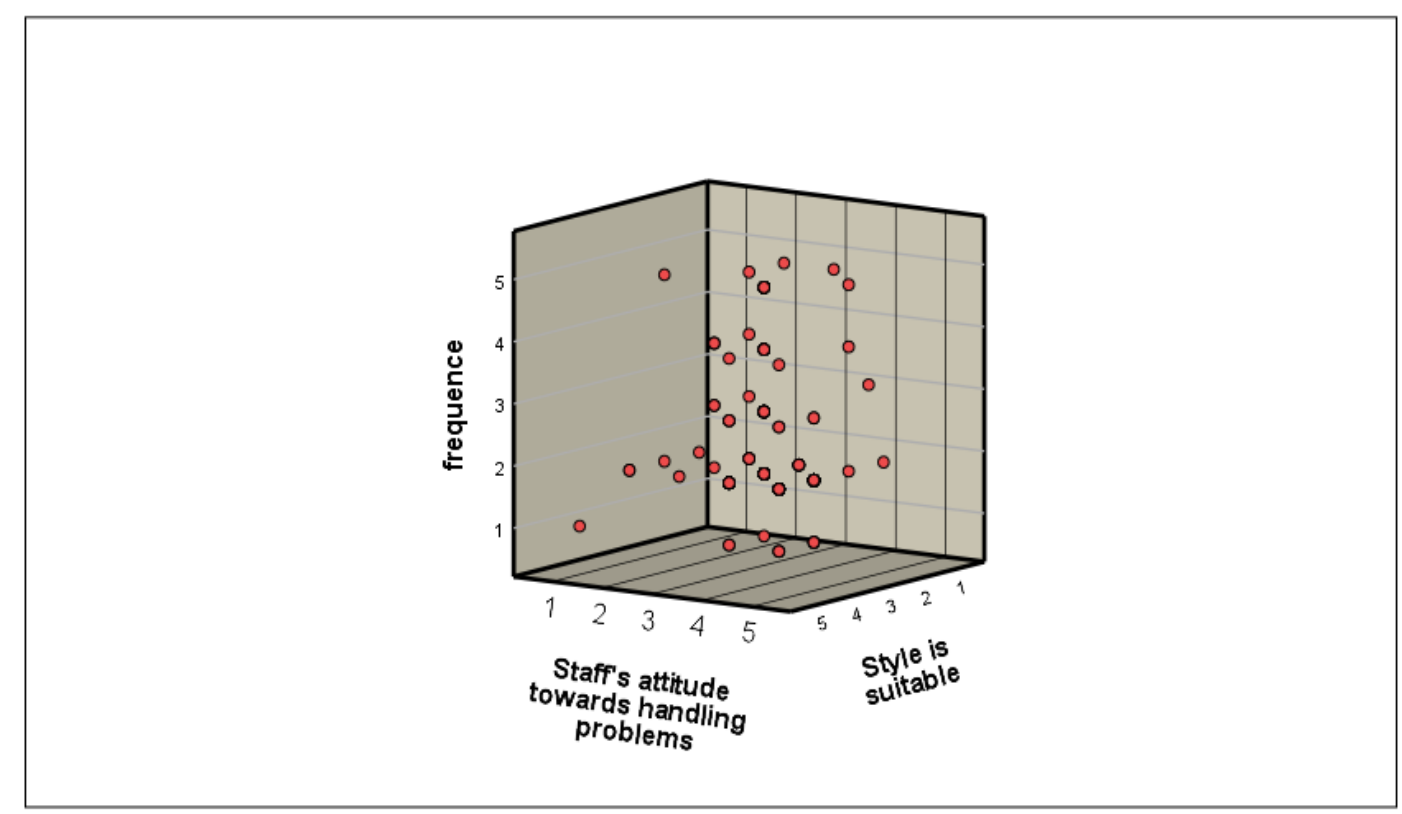

Figure 2. Data visualization of K-Means cluster 
From Figure 2, we can find that the higher the average monthly income of women, the more attention is paid to whether the style of clothing design is suitable for themselves, and the more attention is paid to the service attitude of e-commerce, and the frequency of purchases of clothing is not low.

\subsection{Sentiment Analysis Results}

The sentiment analysis scores were calculated for the 11,864 texts collected in this study, the influencing variables with higher scores are shown in Table 1. The positives scores, negative scores and the sentiment analysis scores as Figure 3. The number of reviews and the sentiment analysis scores as Figure 4.

Table 1 . The number of positives, negatives reviews and the sentiment analysis scores

\begin{tabular}{lcccc}
\hline & style & fashion & service attitude & price \\
\hline Number of positives & 2,732 & 1,423 & 891 & 223 \\
Number of negatives & 1,124 & 682 & 765 & 84 \\
Number of reviews & 3,856 & 2,105 & 1,656 & 307 \\
Sentiment analysis scores & 1,608 & 741 & 126 & 139 \\
\hline
\end{tabular}

From Table 1, Figure 3, and Figure 4, we can find that in customer reviews, the most mentioned variables are clothing style, fashion, service attitude and price. There are 2,732 reviews that give positive evaluations to clothing style, and 1,124 reviews that give negative evaluations. The number of reviews on clothing style is 3,856 and the sentiment analysis scored 1,608 points, all ranking first. There are 1,423 reviews that give positive evaluations to clothing fashion, and 682 reviews that give negative evaluations. The number of reviews on clothing fashion is 2,105 and the sentiment analysis scored 741 points, all ranking second. There are 891 reviews that give positive evaluations to the service attitude, and 765 reviews that give negative evaluations. The number of reviews on service attitude is 1,656 , ranking third, but the sentiment analysis score of service attitude is only 126 points, ranking fourth. There are 223 reviews that give positive evaluations to the price, and 84 reviews that give negative evaluations. The number of reviews on price is only 307 , ranking fourth, but the sentiment analysis score of prices is 139 points, ranking third.

The reason is that although there are few customer reviews on the price, the number of positive reviews far exceeds the number of negative reviews. There are many customer reviews on service attitudes, the number of reviews that give positive and negative reviews very close, so the sentiment analysis score is low. In other words, for small and mediumsized clothing e-commerce enterprises, it is important to strengthen the education and training of employees, especially the service attitude of employees to deal with problems. Improving the service attitude will quickly improve customers' evaluation of small clothing e-commerce enterprise. 


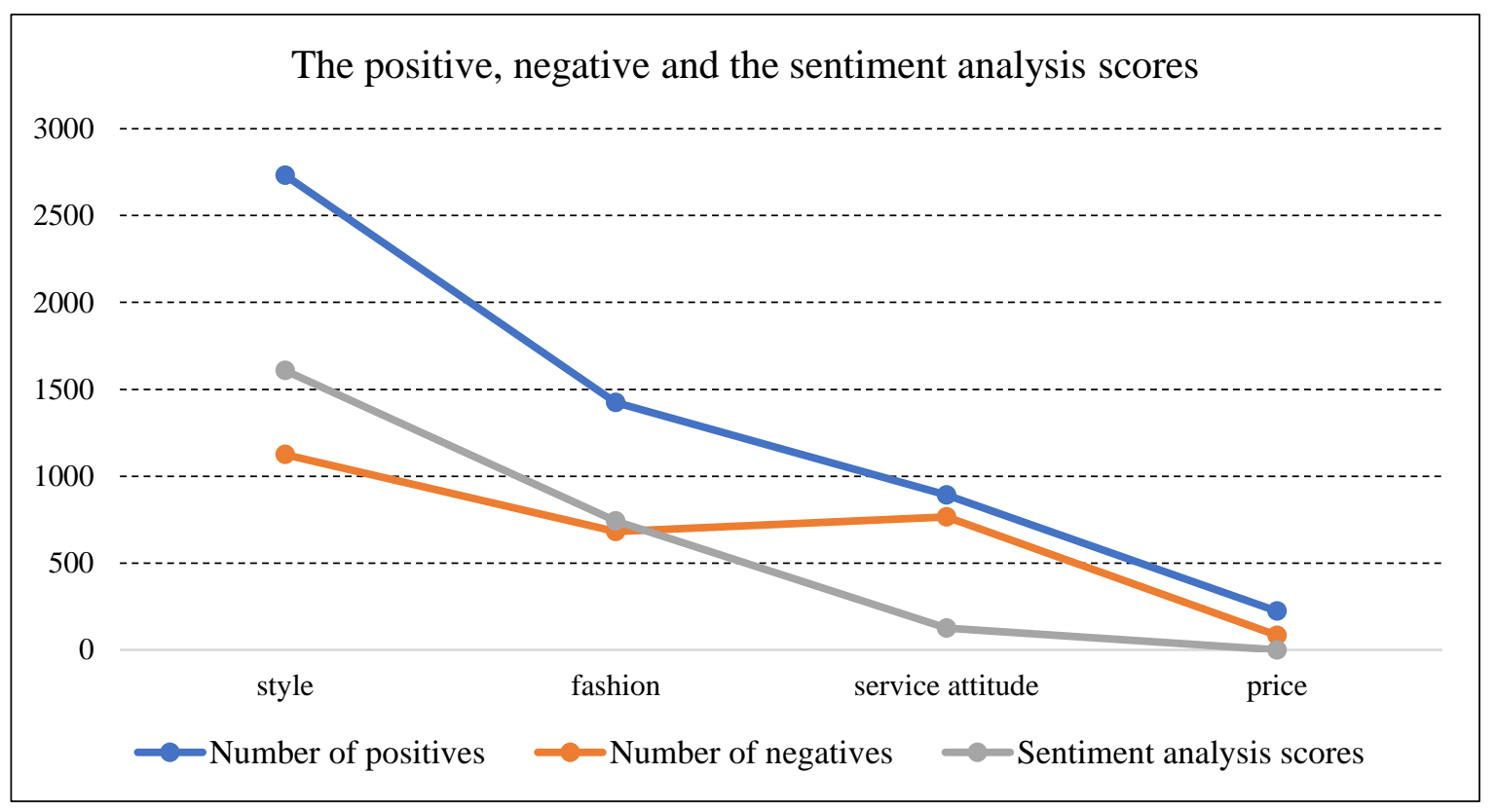

Figure 3. The positive, negative and the sentiment analysis scores

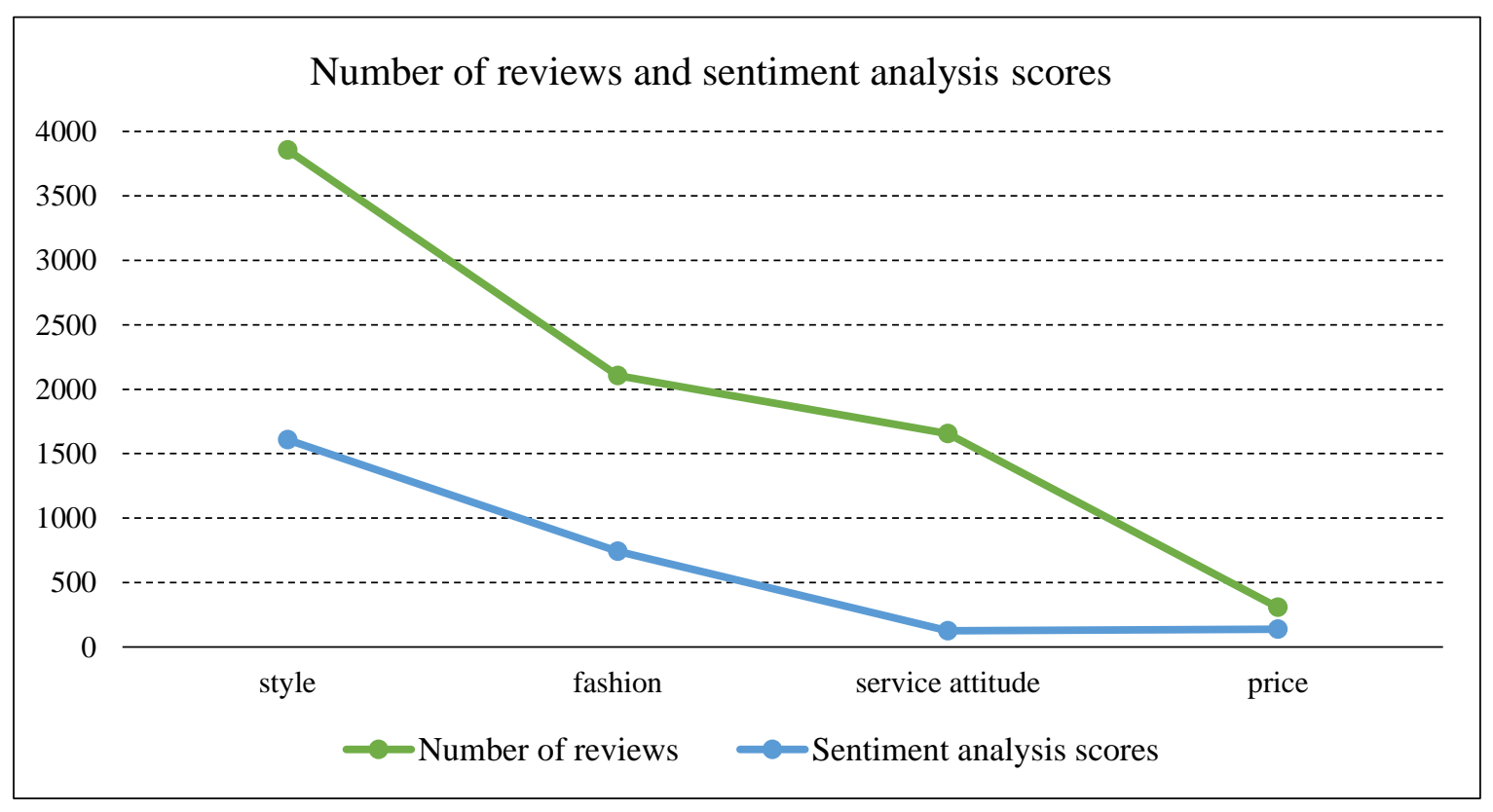

Figure 4. The number of reviews and the sentiment analysis scores

\subsection{Precision Marketing Suggestions}

Based on the results of decision tree analysis, this paper believes that precision marketing of the small and medium-sized clothing e-commerce enterprises should first focus on consumer groups and adopt different marketing strategies to push different styles of clothing according to different types of consumers. Secondly, pay attention to the staff service training of small and medium-sized clothing e-commerce enterprises. Finally, the attractive fashion design of clothing is also important. Therefore, this paper puts forward reasonable suggestions from four aspects of marketing object, marketing strategies, enterprise training and clothing design. 


\subsubsection{Gender Recommendation Strategies}

Based on the different needs and psychological choices of different genders, this study suggests that gender recommendation strategies should be adopted. Since $68 \%$ of men buy clothes because of the change of seasons, it is necessary for male consumers to push the advertising and discount of seasonal clothes during the season change. For female consumers, it is necessary to analyze the purchase tendency and clothing style, and construct the analogy recommendation system for similar users.

\subsubsection{Personalized Recommendation Strategies}

The style fit of clothing is the first consideration for consumers' purchase of clothing. This paper thinks that consumers should be effectively classified according to the style and favorite design of the clothing purchased, such as leisure style, national dress style, lady style, cowboy style, hippie style, and other styles, as well as stripe style, dot style, elegant style, colorful design, etc., to explore the design patterns that consumers like in the same style. Then make personalized recommendations based on the types of consumers.

From the data, there are different categories of male user groups. Most men tend to choose comfortable and practical formal clothes, while some men are interested in fashion and don't care whether the clothing is comfortable and practical. The age of this group is generally younger, the number of people is fewer, and the style is different from the general trend style of men. Therefore, fashionable style clothing should be recommended to young men.

The higher the average monthly income of women, the more attention is paid to whether the style of clothing design is suitable for themselves, and the more attention is paid to the service attitude of e-commerce enterprises. The precise marketing of big data platform should classify consumers, collect favorite clothing design, establish consumer behavior model and build the analogy recommendation system for similar users by comparing the same type of consumers who buy the same style clothing, collecting favorite clothing design and shopping preferences. In this way, we can use the established system to make the same personalized recommendation to the same type of consumers who like the same style.

\subsubsection{Differentiated Marketing Strategies}

Based on $68 \%$ of men will choose to buy clothes due to seasonal changes, but only $31 \%$ of women buy clothes because of the seasonal change, so we believe that we should pay attention to the seasonal differences of marketing, i.e., the differentiated marketing strategies should be carried out based on changes in time. Each season in the year, differentiated marketing recommendation should be carried out. Warm jackets, coats, etc. are recommended in winter and polo shirts and silk shirts, etc. are recommended in summer.

\subsubsection{Enterprise Training and Clothing Design Strategies}

The fit of clothing style is the most important factor affecting the purchase. The higher the average monthly income of women, the more attention is paid to whether the style of clothing design is suitable for themselves, and the more attention is paid to the service attitude of e-commerce enterprise. There are two things important. Firstly, the small and medium-sized clothing e-commerce enterprises should strengthen the connection between their fashion designers and international fashion trends. When the clothing sold by the ecommerce enterprise is combined with international fashion trends, and the clothing sold in various styles, we can carry out various differentiated marketing strategies according to the different type of consumers. Secondly, the small and medium-sized clothing e-commerce enterprises should pay attention to the staff service training of small and medium-sized 
clothing e-commerce enterprises, and pay special attention to service attitude and service speed.

\section{Conclusions and Future Research}

\subsection{Conclusions}

In this paper, we attempt to explore the factors that affect the sales of e-commerce platforms through data, establish related big data analysis methods, and conduct empirical research with small and medium-sized clothing e-commerce enterprises on Tmall as an example. According to the results of big data analysis, precision marketing strategies are proposed for small and medium-sized clothing e-commerce enterprises. We have proposed the gender recommendation strategies, personalized recommendation strategies, differentiated marketing strategies, enterprise training and clothing design strategies. These precision marketing strategies are feasible and practical.

For male consumers, we suggest to push the advertising and discount of seasonal clothes during the season change. The style fit of clothing is the first consideration for consumers' purchase of clothing. We suggest that consumers should be effectively classified according to the style and favorite design of the clothing purchased, to explore the design patterns that consumers like in the same style and make personalized recommendations. The higher the average monthly income of women, the more attention is paid to whether the style of clothing design is suitable for themselves, and the more attention is paid to the service attitude of e-commerce enterprise. From big data of the customer reviews, the number of reviews on service attitude is ranking third, but the sentiment analysis score of service attitude is ranking fourth. The reason is that the number of reviews that give positive and negative reviews are very close, so the sentiment analysis score is low. So we suggest that the small and medium-sized clothing e-commerce enterprises pay attention to the staff training of service attitude, we also suggest that they should strengthen the connection between their fashion designers and international fashion trends.

Today, the application of big data in marketing is in the development stage, involving a wide range of fields and complex technology. However, with the continuous breakthroughs in data mining technology, the big data precision marketing strategy of ecommerce enterprise will continue to develop.

\subsection{Future Research}

In the process of this research, we need to establish the subject vocabulary database, attribute vocabulary database, negative vocabulary database, neutral vocabulary database, and evaluation vocabulary database, but we think that they are not complete. The sentiment analysis algorithm we also think that it is not perfect, and sometimes it still needs to be judged manually and given scores to operate, so the sentiment analysis technology still has great improvements. For example, the use of fuzzy algorithms, restricted growth grid neural network clustering model, adaptive restricted growth grid neural network clustering methods to improve emotional analysis technology etc.

In the foreseeable future, the analysis of e-commerce big data will rely on artificial intelligence, and interact with consumers through speech and voice recognition software. The distributive systems of many e-commerce enterprises will interface with virtual reality software or augmented reality software, give customer experiences to attract customers who purchase products online to experience the customer. Therefore, the relevant data will be more diverse and the amount of data will be larger, the relevant big data analysis methods will be more complex and the required technology will be higher-end. 


\section{References}

1. Beyer, M. A., \& Laney, D. (2012). The importance of 'Big Data': A definition.

2. Blackwell, D. R., Paul, W. M., \& James, F. E. (2001). Consumer behavior, 9th Ed, Ohio: Mike Roche, 48-53.

3. Brynjolfsson, E., Hitt, L. M., \& Kim, H. H. (2011). Strength in numbers: How does data-driven decision-making affect firm performance? International Conference on Information Systems, ICIS 2011 (2011) 1 541-558.

4. Camilleri, M. A. (2018). The promotion of responsible tourism management through digital media, Tourism Planning \& Development, 15(6), 653-671.

5. Del Rowe, S. (2016). Beyond the three V's of big data: There are as many as five V's, but one trumps all of them. CRM Magazine, 20(6), 29-31.

6. Hatzivassiloglou, V., \& McKeown, K. R. (1997). Predicting the semantic orientation of adjectives. In Proceedings of ACL-97, 174-181.

7. Hirzel, M., Andrade, H., Gedik, B., Jacques-Silva, G., Khandekar, R., \& Kumar, V., Mendell, M., Nasgaad, H., Schneider, S., Soule, R., \& Wu, K. L. (2013). IBM streams processing language: Analyzing big data in motion. Ibm Journal of Research \& Development, 57(3), 7:1-7:11.

8. Kitchen, P. J., Kim, I., \& Schultz, D. E. (2008). Integrated marketing communications: practice leads theory. Journal of advertising research, 48(4), 531-546.

9. Kotler, P. (2000). Marketing management: Millennium Edition, International Edition.

10. Kusnetzky, D. (2018). Big data and business intelligence: What's the difference? https://www.dataversity.net/big-data-business-intelligence-whats-difference/

11. Laney, D. (2001). 3D data management: Controlling data volume, velocity, and variety. META Delta Application Delivery Strategies, ADS 6, Feb. 01, 949.

12. Reichheld, F. F. \& Schefter, P. (1996). The loyalty effect: The hidden force behind growth, profits, and lasting value. Boston: Harvard Business School Press.

13. Reichheld, F. F. \& Schefter, P. (2000). Your secret weapon on the Web. Harvard Business Review, 78(4), 105-113.

14. Riloff, E., \& Wieber, J. M. (2003). Learning extraction patterns for subjective expressions. Proceedings of the Conference on Empirical Methods in Natural Language Processing (EMNLP-03), 105-112.

15. Rulac, S. E. (1994). Retail real estate in the 21st century: Information technology + time consciousness + unintelligent stores $=$ intelligent shopping? Not! The Journal of Real Estate Research, 9(1), 125-150.

16. Saidali, J., Rahich, H., Tabaa, Y., \& Medouri, A. (2019). The combination between big data and marketing strategies to gain valuable business insights for better production success. Procedia Manufacturing, 32, 1017-1023.

17. Schiffman, L., \& Kanuk, L. (2014). Consumer behavior $\left(10^{\text {th }}\right.$ ed.). Cape Town, South Africa: Pearson Education.

18. Yao, Y. Y. (2012). An outline of a theory of three-way decisions. International Conference on Rough Sets and Current Trends in Computing Springer, Berlin, Heidelberg.

19. You, Z., Si, Y. W., Zhang, D. F., Zeng, X. X., Leung, S. C. H., \& Li, T. (2015). A decision-making framework for precision marketing. Expert Systems with Applications, 7, 3357-3367. 